\title{
Erratum: Nonleptonic weak decays of bottom baryons \\ [Phys. Rev. D 56, 2799 (1997)]
}

\author{
Hai-Yang Cheng
}

(Q) (Received 20 March 2019; published 1 April 2019)

DOI: 10.1103/PhysRevD.99.079901

There are typographical errors in the first line of Eq. (B2), which should read

$$
\Gamma\left(1 / 2^{+} \rightarrow 3 / 2^{+}+P\right)=\frac{p_{c}^{3}}{12 \pi}\left\{\frac{\left(m_{i}+m_{f}\right)^{2}-m_{P}^{2}}{m_{f}^{2}}|C|^{2}+\frac{\left(m_{i}-m_{f}\right)^{2}-m_{P}^{2}}{m_{f}^{2}}|D|^{2}\right\} .
$$

The numerical results in Table III remain unchanged as they were calculated using the above correct equation. There is one typographical error in the first line in Eq. (B3), which should read

$$
\Gamma\left(1 / 2^{+} \rightarrow 1 / 2^{+}+V\right)=\frac{p_{c}}{4 \pi} \frac{E_{f}+m_{f}}{m_{i}}\left[2\left(|S|^{2}+\left|P_{2}\right|^{2}\right)+\frac{E_{V}^{2}}{m_{V}^{2}}\left(|S+D|^{2}+\left|P_{1}\right|^{2}\right)\right] .
$$

As a consequence, the numerical results in Table II for the decay rate $\Gamma$ of $1 / 2^{+} \rightarrow 1 / 2^{+}+V$ should be multiplied by a factor of 2 . For example, $\Gamma\left(\Lambda_{b}^{0} \rightarrow \rho^{-} \Lambda_{c}^{+}\right)$should read $0.88 a_{1}^{2}$ instead of $0.44 a_{1}^{2}$. 